\title{
A Comparative study of four Persian versions of sleep questionnaires for screening obstructive sleep apnea syndrome (OSAS)
}

\author{
Fatemeh Kashaninasab ${ }^{1}$, Kaveh Alavi ${ }^{2}$, Mohammad Farhadi ${ }^{3}$, Mansour Salehi $^{2}$, Mir Farhad Ghaleh Bandi²*
}

Received: 25 Sep 2016 Published: 23 Dec 2017

\begin{abstract}
Background: Diagnosis of obstructive sleep apnea syndrome (OSAS) is valuable, but it is time-consuming and expensive. Appropriate screening instruments help clinicians select high-risk individuals for further investigations. In the present study, we compared 4 popular instruments used in screening OSAS including Berlin, STOP, STOP-BANG questionnaires, and Epworth Sleepiness Scale (ESS).

Methods: A total of 250 individuals, who referred to Sleep Laboratory of Shoorideh Hospital (Tehran, Iran) for polysomnography during May 2015 to November 2015, were recruited for this cross-sectional study. In addition to taking history and physical examination, 4 screening instruments including Berlin, STOP, STOP-BANG questionnaires, and ESS were completed. Diagnosis of OSAS was established using apnea-hypopnea index (AHI) in 3 categories of mild, moderate, and severe.

Results: Severe OSAS was diagnosed in $159(63.6 \%)$, moderate OSAS in $43(17.2 \%)$, and mild OSAS in 41 (16.4\%) of the participants, moreover, AHI was within normal range in the other 7 (2.8\%). To diagnose OSAS with any severity, Berlin questionnaire was a preferable instrument, with a sensitivity of $79.8 \%$ and specificity of $71.4 \%$, considering the cut-point value of 3.5 . In addition, in cases of severe OSAS, Berlin questionnaire showed superiority over other instruments, with a sensitivity of $80.5 \%$ and specificity of $61.5 \%$ using the cut-point value of 3.5 .

Conclusion: None of the 4 instruments are ideal to predict OSAS. However, considering the simplicity and availability of the instruments, Berlin and STOP-BANG questionnaires had maximum diagnostic values that helped us distinguish OSAS and severe OSAS, respectively.
\end{abstract}

Keywords: Sleep questionnaires, Obstructive sleep apnea syndrome, Shoorideh Hospital

Copyright $\odot$ Iran University of Medical Sciences

Cite this article as: Kashaninasab F, Alavi K, Farhadi M, Salehi M, Ghaleh Bandi MF. A Comparative study of four Persian versions of sleep questionnaires for screening obstructive sleep apnea syndrome (OSAS). Med J Islam Repub Iran. 2017 (23 Dec);31:122. https://doi.org/10.14196/mjiri.31.122

\section{Introduction}

One frequent disorder that occurs during sleep is obstructive sleep apnea syndrome (OSAS) that is manifested by frequent upper airway obstructions when the person sleeps. This obstruction causes cessation (apnea) or remarkable decrease (hypopnea) in airflow in spite of respiratory effort (1). The prevalence of OSAS in 30 to 60 year-old adults is around $2 \%$ to $5 \%$ worldwide (2). Obstructive sleep apnea

Corresponding author: Dr Mir Farhad Ghaleh Bandi, ghalehbandi.m@iums.ac.ir

1. Rasoul Akram Hospital, Iran University of Medical Sciences, Tehran, Iran.

2. Mental Health Research Center, Psychiatric Department, Iran University of Medical Sciences, Tehran, Iran.

3. HEAD \& Neck Surgery Department, Research center of Otolaryngology, Rasoul Akram Hospital, Iran University of Medical Sciences, Tehran, Iran. syndrome is more frequent than central apnea (3). Basically, hypercapnia and hypoxemia occur due to obstruction, causing an increase in respiratory effort. However, this respiratory effort is not effective because the airway collapses and the condition remains unchanged until the patient awakens and ends the obstruction; this cycle is repeated during the sleeping time (4-6). Loud snoring is the most

$\uparrow$ What is "already known" in this topic:

Polysomnography is a gold standard to diagnose obstructive sleep apnea syndrome, which is a common sleep disorder. However, it is expensive, time- consuming, and is not easily accessible. A new tool that is less expensive, more available, and more sensitive can be of great help in screening patients with OSAS.

$\rightarrow$ What this article adds:

Berlin and STOP-BANG questionnaires showed more sensitivity and specificity in screening OSAS compared to STOP and ESS. These questionnaires are appropriate alternatives for screening OSAS patients, as they take less time, are cheaper, and more accessible compared to polysomnography. 
common and a classic symptom of OSAS; other nocturnal symptoms include gasping and choking sensations, insomnia, drowsiness, headache, fatigue, and cognitive deficits and confusion during daytime (6). During physical examinations, patients usually seem healthy; however, in some cases, hypertension, enlarged tonsils, high arched hard palate, and other systemic diseases are detectable $(7,8)$. Polysomnography (PSG) is a gold standard diagnostic tool and confirms the diagnosis of OSAS (9). Scientists have applied some questionnaires-based screening tools including Berlin, STOP, STOP-Bang, and Epworth Sleepiness Scale questionnaires, which can be good alternatives to polysomnography.

However, PSG is an expensive tool and is not available everywhere $(10,11)$. The level of the OSAS risk in patients can be determined by the help of Berlin questionnaire. Moreover, STOP questionnaire is a functional tool for screening OSAS in patients who need to undergo a surgery. Similarly, STOP-Bang questionnaire is a more useful tool because it covers body mass index, age, neck circumference, and sex, combined with STOP questionnaires. Furthermore, the Epworth Sleepiness Scale (ESS) questionnaire assesses daytime drowsiness in patients (10-13). Based on our knowledge, the value of these screening tools has not been compared in a previous study in Iran, Therefore, in this research, the value of 4 screening questionnaires including STOP questionnaire (SQ), STOP-BANG questionnaire (SBQ), Epworth Sleepiness Scale (ESS), and Berlin questionnaire (BQ) was compared with that of polysomnography as the standard screening tool for OSA prediction.

\section{Methods}

A total of 250 participants with suspected OSAS, who referred to Sleep Laboratory of Shoorideh hospital in Tehran to undergo polysomnography during May and November 2015, were enrolled in this cross-sectional study. Patients were eligible to participate if they referred for diagnostic or baseline polysomnography to undergo OSAS assessment and if they completed the questionnaires. We excluded those patients with incomplete questionnaires and those without a written consent. In addition, the procedure of the study was explained to the patients, and a written consent was signed by all participants. Finally, the Ethics Committee of Iran University of Medical Sciences approved the study protocol.

\section{Participants and procedures}

A psychiatrist interviewed the enrolled patients and recorded their demographic information, such as age and sex. Patients' weight was measured with the help of a standard portable balance, with a precision of $0.1 \mathrm{~kg}$; and their height was also measured with no shoes on, with a precision of 0.5 $\mathrm{cm}$; finally, their BMI $(\mathrm{kg} / \mathrm{m} 2)$ was calculated. Moreover, history of drugs, well-known medical diseases, and psychiatric disorders was recorded. Then, polysomnography (Medcare Monet Artist, SUP5 type, serial no 0342030024, Netherlands) was performed for the patients. A decrease in airflow that lasts about 10 seconds or more is called apnea. Hypopnea is defined as a reduction in airflow by $\geq 50 \%$ or a $3 \%$ decrease in the level of oxyhemoglobin saturation. Moreover, in hypopnea, an arousal can be determined in EEG (14). AHI is a common and useful index for assessing the severity of OSAS (2). OSAS was diagnosed if AHI was higher than 5 events per hour of sleep with the presence of drowsiness during the daytime or symptoms of sleep disturbances.

The severity of OSAS was calculated by the American Academy of Sleep Medicine guidelines based on the apneahypopnea index (AHI) as severe (AHI > 30), moderate (AHI: 15-30), and mild (AHI: 5-15) (15). In addition, 4 screening tools for selecting individuals under OSAS risk were fulfilled before polysomnography. These tools were Epworth Sleepiness Scale (ESS), STOP-BANG questionnaire, Berlin Questionnaire (BQ), and STOP. BQ has 3 categories: (1) posing questions about snoring, (2) inquiring about daytime sleepiness, (3) and testing the patients' level of blood pressure and weight (11). The patients' truthfulness in answering the questions about snoring was checked with their bed partner(s) or family members. BQ includes 11 questions, which are classified into 3 categories. There are 5 questions in the first category that determine the severity of snoring and its prevalence as well as any reports of apnea given by their bed partner(s) or family member(s).

The 4 questions subcategorized under the second category are about being somnolent and drowsy while driving; and consequently the 2 questions raised in the third domain were about the history of hypertension $(>140 / 90 \mathrm{~mm} \mathrm{Hg}$ ) and BMI of $>30 \mathrm{~kg} / \mathrm{m} 2$.

The overall BQ score was estimated based on the answers to the questions of the 3 categories. Scores obtained from the first and second domains were considered positive if the answers depicted repeated symptoms ( $>3-4$ times/week), while scores for the third categorization was positive in case of a history of hypertension or a BMI of more than 30 $\mathrm{kg} / \mathrm{m} 2$.

A high risk patient for OSAS was defined as a patient with a positive score in at least 2 or more domains $(10,16)$.

Internal consistency for the first and second categories of the BQ in the Persian version was calculated separately. The Cronbach's alpha coefficient for the first category was 0.56 and it was 0.54 for the second category, which was increased to 0.78 by deleting Question 8 . In the test-retest analysis, the intraclass correlation coefficient (ICC) was calculated to be 0.90 (95\% confidence interval: $0.84-0.94)$ (17). STOP questionnaire includes 4 questions as follow:

These 4 questions contain queries about snoring, tiredness, and daytime sleepiness, having difficulty breathing while sleep, and issues with blood pressure.

The STOP questionnaire in the Persian version revealed 0.88 (95\%CI: $0.83-0.91)$ of intraclass correlation coefficient (ICC) (1).

STOP-BANG questionnaire adds 4 additional parameters into the STOP questionnaire, such as BMI (body mass index more than $35 \mathrm{~kg} / \mathrm{m}^{2}$ ), age ( $>50$ years of age), neck circumference of more than $40 \mathrm{~cm}$, and sex (male).

The answers to STOP-BANG and STOP questionnaires were designed within a simple yes/no format and scored from 0 to 4 for STOP questionnaire and 0 to 8 for STOP- 
BANG questionnaire. Both questionnaires score individuals as either high risk or low risk for OSAS.

Those participants giving a yes answer to at least 2 questions in STOP questionnaire and to at least 3 questions in STOP-BANG questionnaire were considered high risk, while those responding yes to minimum of 2 questions in SQ questionnaire and to less than 3 questions in SBQ questionnaire were considered low risk (1). Epworth Sleepiness Scale questionnaire is a self-report questionnaire; the overall ESS score is the aggregation of the scores of 8 questions and is between 0 and 24. Moreover, it measures the general daytime drowsiness.

ESS scoring was divided into $<10$ (low risk for sleepiness) and $\geqslant 10$ (high risk for sleepiness), and Cronbach's alpha coefficient for the Persian version of Epworth Sleepiness Scale in patients indicated by PSG was 0.82 . ICC was calculated to be 0.81 (95\% CI: 0.74-0.86) (18).

\section{Statistical analysis}

Data analysis was performed by SPSS Version 22 for Windows (SPSS Inc., Chicago, IL). Categorical variables were shown as frequency and percentage. Chi square test or Fisher's exact test were used to compare the categorical variables. Independent samples t test was also employed to run a comparison of the mean values of continuous independent variables between the 2 experimental groups. A receiver operating characteristic (ROC) curve was used to show a discriminating value of questionnaires to predict OSAS. The best cut-off value was also determined for each questionnaire to maximize (sensitivity + specificity) the prediction power of OSAS. P- value of 0.05 or less was considered statistically significant.

\section{Results}

A total of 250 participants $(76.0 \%$ males and $24.0 \%$ females) with the mean \pm SD age of $48.1 \pm 12.0$ years (ranging from 19 to 82 years) were evaluated (Table 1). The mean \pm SD AHI score was $44.1 \pm 31.2$ (median: 36.0 , range: one to 181). Based on AHI, 159 participants (63.6\%) suffered from severe OSAS, $43(17.2 \%)$ from moderate OSAS, and $41(16.4 \%)$ from mild OSAS. In the other 7 persons $(2.8 \%)$, AHI was within normal range (Table 2). The scores for all 4 questionnaires were significantly correlated

Table 1. Patients characteristics at baseline

\begin{tabular}{|c|c|c|}
\hline \multicolumn{3}{|l|}{ Patients characteristics } \\
\hline \multirow[t]{2}{*}{ Sex } & Male & $190(76 \%)$ \\
\hline & Female & $60(24 \%)$ \\
\hline \multirow[t]{2}{*}{ Age (year) } & Mean $\pm \mathrm{SD}$ & $48.1 \pm 12.0$ \\
\hline & Range & $19-82$ \\
\hline \multirow[t]{3}{*}{ Marital status } & Single & $34(13.6 \%)$ \\
\hline & Married & $200(80 \%)$ \\
\hline & Divorced & $16(6.4 \%)$ \\
\hline \multirow[t]{3}{*}{ Education } & Undergraduate & $34(13.6 \%)$ \\
\hline & Diploma & $70(28 \%)$ \\
\hline & College & $146(58.4 \%)$ \\
\hline \multirow[t]{3}{*}{$\mathrm{BMI}\left(\mathrm{kg} / \mathrm{m}^{2}\right)$} & $25-29.9$ & $115(46 \%)$ \\
\hline & $30-34.9$ & $60(24 \%)$ \\
\hline & $>35$ & $37(14.8 \%)$ \\
\hline Neck circumference $(\mathrm{cm})$ & Mean $\pm \mathrm{SD}$ & $40.2 \pm 3.8$ \\
\hline Medication use & & $75(30 \%)$ \\
\hline Smoking & & $68(25.2 \%)$ \\
\hline Opium consumption & & $8(3.2 \%)$ \\
\hline \multirow[t]{10}{*}{ Underlying diseases } & Postnasal drip & $109(43.6 \%)$ \\
\hline & Mood disorders & $88(35.2 \%)$ \\
\hline & Gastro-esophageal reflux & $78(31.2 \%)$ \\
\hline & Leg cramps & $73(29.2 \%)$ \\
\hline & Hypertension & $56(22.4 \%)$ \\
\hline & Diabetes & $34(13.6 \%)$ \\
\hline & Migraine & $34(13.6 \%)$ \\
\hline & Pulmonary diseases & $34(13.6 \%)$ \\
\hline & Cardiovascular diseases & $30(12 \%)$ \\
\hline & Thyroid dysfunction & $25(10 \%)$ \\
\hline \multirow[t]{5}{*}{ Sleep quality satisfaction } & Very satisfied & $10(4 \%)$ \\
\hline & Satisfied & $62(24.8 \%)$ \\
\hline & Partially satisfied & $87(34.8 \%)$ \\
\hline & Unsatisfied & $65(26 \%)$ \\
\hline & Very unsatisfied & $24(9.6 \%)$ \\
\hline
\end{tabular}

Table 2. Characteristics of different components of Polysomnography

\begin{tabular}{|c|c|c|c|}
\hline Item & Mean \pm SD & Median & Range \\
\hline Total sleep time, $\min$ & $379.1 \pm 81.4$ & 380.5 & $90-553$ \\
\hline Sleep latency, min & $16.9 \pm 24.6$ & 8.0 & $5.0-190.5$ \\
\hline Sleep efficiency, $\%$ & $84.3 \pm 14.2$ & 88.4 & $15.2-100.0$ \\
\hline REM period time, min & $53.2 \pm 40.5$ & 48.0 & $0-300.0$ \\
\hline REM latency, min & $141.8 \pm 83.5$ & 131.5 & $0-398.0$ \\
\hline Events (apnea, hypopnea) & $271.2 \pm 203.2$ & 220.0 & $6.0-1136.0$ \\
\hline Mean oxygen saturation, $\%$ & $92.1 \pm 4.5$ & 93.0 & $61.4-98.0$ \\
\hline
\end{tabular}


Table 3. Correlation between the scores for all study questionnaires

\begin{tabular}{lcccc}
\hline & Berlin's questionnaire & STOP questionnaire & STOP-BANG questionnaire & AHI \\
\hline ESS & $0.335^{* *}$ & $0.277^{* *}$ & $0.209 * *$ & $0.207 * *$ \\
Berlin's questionnaire & - & $0.762 * *$ & $0.656 * *$ & $0.484 * *$ \\
STOP questionnaire & - & - & $0.793 * *$ & $0.406 * *$ \\
STOP-BANG questionnaire & - & - & - & $0.479 * *$ \\
\hline$* *$ P-value $<0.001$ & & &
\end{tabular}

** P-value $<0.001$

Table 4. Correlation between the scores for study questionnaires and components of Polysomnography

\begin{tabular}{lcccc}
\hline Item & ESS & Berlin's questionnaire & STOP questionnaire & STOP-BANG questionnaire \\
\hline Total sleep time & 0.039 & 0.078 & 0.034 & -0.023 \\
Sleep latency & 0.059 & -0.005 & -0.034 & 0.021 \\
Sleep quality & 0.012 & 0.082 & 0.094 & 0.015 \\
REM period time & 0.032 & -0.032 & -0.016 & -0.051 \\
REM latency & -0.007 & 0.096 & 0.076 & 0.073 \\
Events (apnea, hypopnea) & $0.197 *$ & $0.473 * *$ & $0.376 * *$ & $0.432 * *$ \\
Mean oxygen saturation & $-0.234 * *$ & $-0.274 * *$ & $-0.289 * *$ & $-0.385 * *$ \\
\hline
\end{tabular}

* P-value $<0.05$

$* *$ P-value $<0.001$

with AHI score. Furthermore, the total scores for questionnaires were also correlated with each other; however, the correlation between ESS score and other questionnaires was partially weak (Table 3 ). Among different components of polysomnographic evaluation, the scores for sleep-related events including apnea and hypopnea were positive, and oxygen saturation was inversely correlated with the scores for ESS, Berlin questionnaire, STOP-BANG, and STOP questionnaires (Table 4). Overall, the area under the ROC (AUC) analysis (Fig. 1) showed that ESS scores could not effectively predict OSAS. Also, considering OSAS diagnosis on $\mathrm{AHI} \geq 5$, Berlin questionnaire had the highest AUC to discriminate OSAS. The ROC curve analysis also indicated that the estimated cut-off points yielded sensitivity or specificity higher than $85.0 \%$ for none of the questionnaires, while at the cut-off value of 3.5 , the BQ questionnaire had a sensitivity of $79.8 \%$ and a specificity of $71.4 \%$ to discriminate OSAS from a normal condition (Table 4). To assess moderate to severe OSA (AHI $\geq 15)$, ESS could not predict OSAS, while BQ had the highest AUC, indicating the highest value to predict moderate to severe OSAS among the 4 study questionnaires. At the cut-off point of 4.5 , BQ had a sensitivity of $72.8 \%$ and a specificity of $75.0 \%$ to diagnose moderate to severe OSAS, while at the cut-off value of 3.5, SBQ revealed sensitivity and specificity of $74.8 \%$ and $75.0 \%$, respectively (Table 5 and Fig. 2). To diagnose severe OSAS (AHI $\geq 30$ ), ESS was unable to predict this clinical condition. Among other 4 questionnaires and according to the ROC analysis, the diagnostic superiority was related to SBQ with the cut-off value of 3.5 , revealing a sensitivity of $80.5 \%$ and a specificity of $61.5 \%$. The best cut-off value for BQ to discriminate severe OSAS was 5.5 , indicating a sensitivity of $63.5 \%$ and a specificity of $76.9 \%$ (Table 5 and Fig. 3). For more details see Table 6.

\section{Discussion}

OSAS is an important sleep disorder because of its correlation with some systemic diseases; it affects $2 \%$ to $4 \%$ of the general population worldwide (19). In this practice, we compared 4 established sleep questionnaires including STOP-BANG, STOP, ESS, and Berlin questionnaires to evaluate the predictive value of these questionnaires to diagnose OSAS. Then, the values of these questionnaires were compared to AHI, serving with the title of gold standard diagnosis of OSAS. In some studies, STOP-BANG and STOP questionnaires were applied as they were the preoperative determining tools in participants under surgery to stratify patients with unrecognized OSAS to prevent intra-

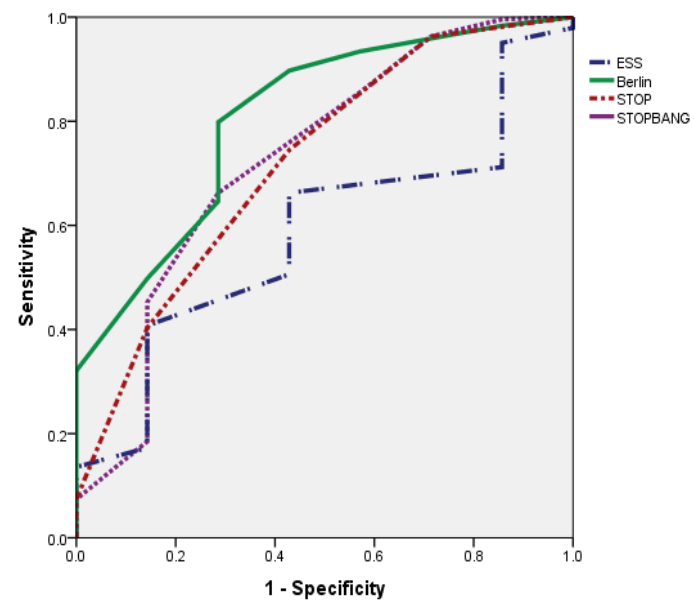

Fig. 1. Area under the ROC curve to predict OSA

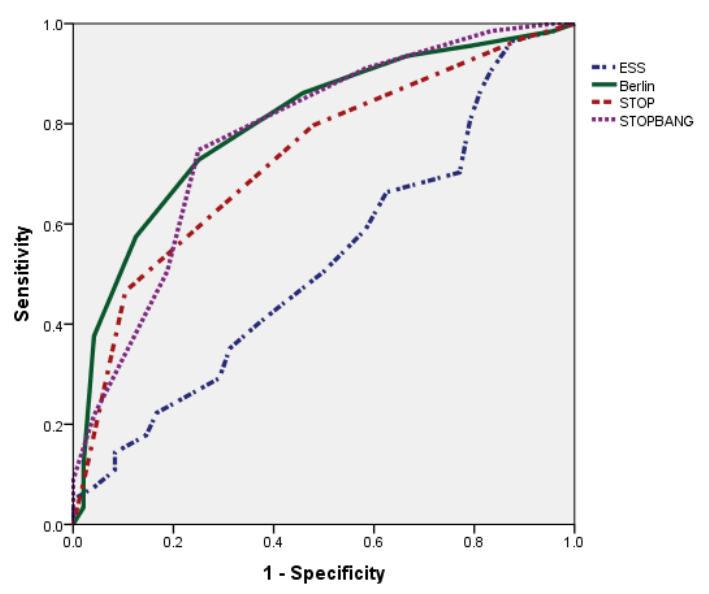

Fig. 2. Area under the ROC curve to predict moderate to severe OSA 


\begin{tabular}{|c|c|c|c|}
\hline Questionnaire & AUC & P-value & $95 \%$ Confidence Interval \\
\hline \multicolumn{4}{|l|}{$\mathrm{AHI} \geq 5$} \\
\hline ESS & 0.585 & 0.444 & $0.403-0.767$ \\
\hline Berlin's questionnaire & 0.808 & 0.006 & $0.648-0.967$ \\
\hline STOP questionnaire & 0.723 & 0.045 & $0.525-0.920$ \\
\hline STOP-BANG questionnaire & 0.728 & 0.040 & $0.514-0.941$ \\
\hline \multicolumn{4}{|l|}{$\mathrm{AHI} \geq 15$} \\
\hline ESS & 0.525 & 0.595 & $0.434-0.615$ \\
\hline Berlin's questionnaire & 0.800 & $<0.001$ & $0.733-0.868$ \\
\hline STOP questionnaire & 0.731 & $<0.001$ & $0.656-0.806$ \\
\hline STOP-BANG questionnaire & 0.777 & $<0.001$ & $0.701-0.853$ \\
\hline
\end{tabular}

Table 6. The results of ESS, Berlin, STOP and STOP-Bang questionnaires for the prediction of OSA

\begin{tabular}{|c|c|c|c|c|c|}
\hline & & ESS & Berlin & STOP & STOP-Bang \\
\hline \multirow[t]{6}{*}{$\mathrm{AHI} \geq 5$} & Sensitivity & 40.7 & 79.8 & 74.5 & 66.3 \\
\hline & Specificity & 85.7 & 71.4 & 57.1 & 28.6 \\
\hline & PPV & 99 & 99 & 98.4 & 98.8 \\
\hline & NPV & 4 & 9.3 & 6.1 & 5.7 \\
\hline & $\mathrm{LR}+$ & $2.8(0.46-17.56)$ & $2.79(0.86-9.01)$ & $1.74(0.74-4.09)$ & $0.93(0.58-1.5)$ \\
\hline & LR- & $0.69(0.5-0.95)$ & $0.28(0.17-0.48)$ & $0.45(0.23-0.88)$ & $1.18(0.36-3.85)$ \\
\hline \multirow[t]{6}{*}{$\mathrm{AHI} \geq 15$} & Sensitivity & 17.8 & 72.8 & 46.5 & 74.8 \\
\hline & Specificity & 85.4 & 75 & 89.6 & 75 \\
\hline & PPV & 83.7 & 92.5 & 94.9 & 92.6 \\
\hline & NPV & 19.8 & 32.8 & 28.5 & 41.4 \\
\hline & $\mathrm{LR}+$ & $1.22(0.58-2.57)$ & $2.91(1.77-4.79)$ & $4.47(1.92-10.39)$ & $2.92(1.82-4.92)$ \\
\hline & LR- & $0.96(0.84-1.1)$ & $0.36(0.27-0.48)$ & $0.6(0.5-0.7)$ & $0.34(0.25-0.45)$ \\
\hline \multirow[t]{6}{*}{$\mathrm{AHI} \geq 30$} & Sensitivity & 15.7 & 63.5 & 83 & 80.5 \\
\hline & Specificity & 91.2 & 76.9 & 42.9 & 61.5 \\
\hline & PPV & 75.8 & 82.8 & 85.9 & 78.5 \\
\hline & NPV & 38.2 & 54.7 & 51 & 64.4 \\
\hline & $\mathrm{LR}+$ & $1.78(0.84-3.79)$ & $2.75(1.86-4.07)$ & $1.45(0.85-9.58)$ & $2.09(1.60-2.74)$ \\
\hline & LR- & $0.92(0.84-1.01)$ & $0.47(0.38-0.6)$ & $0.4(0.88-1)$ & $0.32(0.22-0.45)$ \\
\hline
\end{tabular}
predictive value; PPV; positive predictive value.

or postsurgery complications related to $\operatorname{OSAS}(11,12$, and 20). In the current practice, Berlin questionnaire showed the highest sensitivity to OSAS diagnosis, however, regarding severe OSAS, the highest sensitivity was achieved using a STOP - BANG questionnaire. The best diagnostic cutoff point for Berlin and STOP-BANG questionnaires was 3.5 to predict OSAS and severe OSAS, respectively. However, in several cut-off values, the questionnaires did not reveal a sensitivity or specificity higher than $85 \%$ to predict OSAS or severe OSAS. In a similar study, STOP, STOP-

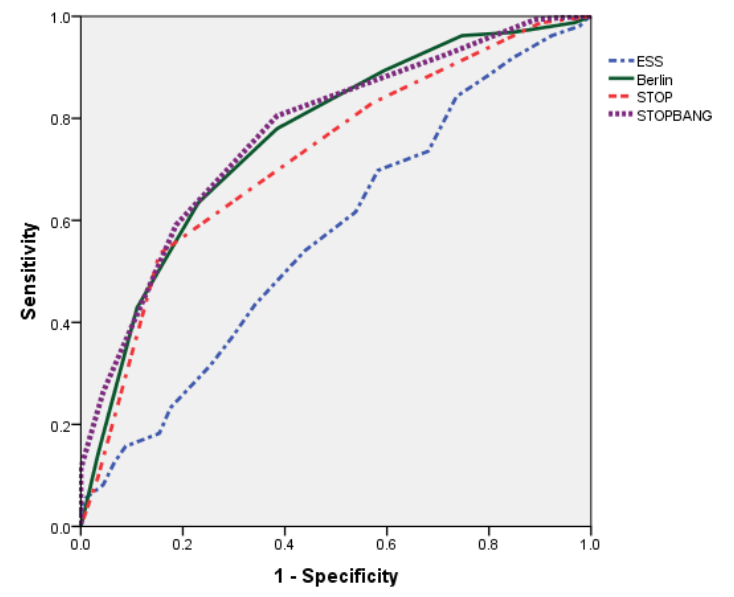

Fig. 3. Area under the ROC curve to predict severe OSA
Bang, and Berlin questionnaires identified more individuals at cut-offs of $\mathrm{AHI} \geqslant 5(91.67 \%, 97.55 \%$, and $95.07 \%$, respectively), $\geqslant 15(94.35 \%, 97.74 \%$, and $95.48 \%$, respectively), and $\geqslant 30(95.95 \%, 98.65 \%$, and $97.3 \%$, respectively). In this cut-off (3.5), the cooperation of the Bang part to the STOP questionnaire increased the sensitivity of the questionnaire in diagnosing OSAS. However, the specificity of these questionnaires was very low at the same cutoffs. Generally, in a healthy population, an ideal diagnostic test is to have an almost high specificity to reduce false positives and sufficient sensitivity.

On the other hand, an acceptable diagnostic test within the population with high pretest possibility of illness shall possess higher sensitivity, while keeping high specificity (11).

Recent practices indicated high sensitivity of STOPBang questionnaire in diagnosing OSAS and moderate and severe OSAS (93\% and $100 \%$, respectively), whereas at the same cut-off point of STOP-Bang questionnaire (score of $\geqslant 3$ ) specificity stood at $47 \%$ and $37 \%$ for moderate and severe OSAS, respectively, indicating rather high false-positive amounts (12).

In a study, Silva et al. compared the 4 screening tools and revealed that the sensitivity of STOP-Bang questionnaire for screening severe and moderate-to-severe sleep-disordered breathing (SDB) $(87.0 \%$ and $70.4 \%$, respectively) was higher than ESS and STOP questionnaire. However, they emphasized that STOP-Bang and STOP questionnaires had the advantage of being easily scored. Moreover, these were considered the most accurate questionnaires for 
determining OSAS in patients who needed a surgery (21). Ahmadi et al. evaluated 130 patients who referred to sleep clinic using RDI (respiratory disturbance index) tool. They revealed $26.2 \%$ respiratory disturbance ( $\mathrm{RDI}>10$ ) among the patients, while $58.5 \%$ of the patients were at a higher risk to sleep apnea using a Berlin questionnaire with $62 \%$ sensitivity and $43 \%$ specificity (22). Such discrepancy between our results and those of the mentioned studies may be due to the use of RDI rather than AHI at a higher cut-off (ie, >10) and patients' selection, as those studies prescreened the patients to check their snoring, its frequency, daytime sleepiness or fatigue, history of high blood pressure, or obesity (11). Compared to other questionnaires, ESS had the lowest sensitivity to anticipate OSAS, moderate-to-severe OSAS, and severe OSAS in our experience. In line with our survey, another study compared ESS, STOP, and STOP-Bang questionnaires and revealed that ESS had the lowest sensitivity for both moderate-to-severe and severe OSAS (39\% and $46.1 \%$, respectively) compared to STOP-Bang and STOP questionnaires (12). Harmoniously, a research by El-Sayed indicated acceptable sensitivity of STOP and STOP-Bang. Moreover, Berlin questionnaires had maximum sensitivity and minimum specificity for the diagnosis of OSAS, while ESS showed low sensitivity and high specificity for the diagnosis of OSAS (10). This may be related to the fact that ESS is a classic questionnaire to determine the level of subjective excessive drowsiness during the day (23), which is a diagnostic criterion for OSAS but can occur secondary to multiple causes other than OSAS. Furthermore, another study indicated that ESS cannot differentiate between simple snore and patients with OSAS accurately (24). A key strength of this study was that full night attended diagnostic polysomnography was performed for all patients, providing the gold standard against which the results of the questionnaires were compared. The questionnaires were answered prior to PSG, which were scored by a sleep specialist who did not know the results of the questioners in advance or other clinical information about the patients to rule out any influence of the polysomnography over the results of the questionnaires. Finally, although some studies suggested both STOP and STOP-Bang questionnaires as the most accurate questionnaires for OSAS screening in surgical patients $(11,12,20$, 21 , the sensitivity of STOP, STOP-Bang, and Berlin questionnaires in this study was at the expense of their specificity. Thus, these questionnaires were able to identify highrisk patients for OSAS, without excluding those at low risk. The most important limitation of this study was a small number of healthy samples, so the specificity and negative predictive value of the test were not highly reliable. One of the weak points of the current study was its unicentric cross-sectional nature, so we could not generalize the results of the study to all Iranian populations, and thus more multicenter studies are required to confirm the results reported here.

\section{Conclusion}

This study revealed that BQ and SBQ questionnaires have the highest value to discriminate OSAS and severe OSAS from normal conditions; however, ESS has not enough power to diagnose OSAS or predict the severity of OSAS.

\section{Acknowledgment}

We would like to thank all staff of the sleep laboratory in Shoorideh hospital and Nour Sleep Disorders Clinic, without whom we could not complete this research.

\section{Conflict of Interests}

The authors declare that they have no competing interests.

\section{References}

1. Sadeghniiat-Haghighi K, Montazeri A, Khajeh-Mehrizi A, Ghajarzadeh M, Alemohammad ZB, Aminian O, et al. The STOP-BANG questionnaire: reliability and validity of the Persian version in sleep clinic population. Qual Life Res. 2015;24(8):2025-30.

2. Sezavar SH, Hajsadeghi S, Hejrati M, Ghaleh Bandi MF. Left Ventricular Mass index and Pulmonary Artery Pressure in Patients with the Obstructive Sleep Apnea Syndrome. J Tehran Heart Cent. 2016;11(1):11-14.

3. Caples SM, Gami AS, Somers VK. Obstructive sleep apnea. Ann Intern Med. 2005;142(3):187-197.

4. Eckert Danny J, Malhotra A. Pathophysiology of Adult Obstructive Sleep Apnea. Proc Am Thorac Soc. 2008;5(2):144-153.

5. Boynton G, Vahabzadeh A, Hammoud S, Ruzicka DL, Chervin RD. Validation of the STOP-BANG questionnaire among patients referred for suspected obstructive sleep apnea. J Sleep Disord Treat Care. 2013;2(4).

6. Patil SP, Schneider H, Schwartz AR, Smith PL.Adult Obstructive Sleep Apnea: Pathophysiology and diagnosis. Chest. 2007; 132(1):325-337.

7. Badr MS, Toiber F, Skatrud JB, Dempsey J. Pharyngeal narrowing/occlusion during central sleep apnea. J Appl Physiol. 1995; 78(5):180615 .

8. Sadock B, Sadock V, Ruiz P. Kaplan and Sadock's comprehensive textbook of psychiatry. 9th ed: Lippincott Williams \& Wilkins 2009. 2157 9p.

9. Gozal D, Kheirandish-Gozal L. Cardiovascular morbidity in obstructive sleep apnea: oxidative stress, inflammation, and much more. Am J Respir Crit Care Med. 2008;177(4):369-75.

10. El-Sayed IH. Comparison of four sleep questionnaires for screening obstructive sleep apnea. Egyptian Journal of Chest Diseases and Tuberculosis. 2012;61(4):433-41.

11. Abrishami A, Khajehdehi A, Chung F. A systematic review of screening questionnaires for obstructive sleep apnea, Can $J$ Anaesth 2010;57(5):423-38.

12. Chung F, Yegneswaran B, Liao P, Chung SA, Vairavanathan S, Islam S, et al. STOP questionnaire: a tool to screen patients for obstructive sleep apnea. Anesthesiology. 2008;108(5):812-21.

13. Netzer NC, Hoegel JJ, Loube D, Netzer CM, Hay B, Alvarez-Sala R , et al. Prevalence of Symptoms and Risk of Sleep Apnea in Primary Care. Chest. 2003;124 (4):1406-14.

14. Ghaleh Bandi MF, Naserbakht M, Tabasi A, Marghaiezadeh A, Riazee Esfahani M, Golzarian Z. Obstructive sleep apnea syndrome and non-arteritic anterior ischemic optic neuropathy. Med J Islam Repub Iran. 2015;29(11):300

15. American Academy of Sleep Medicine. International Classification of Sleep Disorders. Diagnostic and Coding Manual. Second Edition. Westchester, Ill: American Academy of Sleep Medicine; 2005.

16. Kuniyoshi FHS, Zellmer MR, Calvin AD, Lopez-Jimenez F, Albuquerque FN, van der Walt C, et al. Diagnostic accuracy of the Berlin Questionnaire in detecting sleep-disordered breathing in patients with a recent myocardial infarction. Chest. 2011;140(5):1192-7.

17. Sadeghniiat-Haghighi K, Montazeri A, Khajeh-Mehrizi A, Aminian O, Rahimi- Golkhandan A, Sedaghat M. The Berlin Questionnaire: Performance of the Persian Version for Measuring Obstructive Sleep Apnea in Sleep Clinic Population. J Sleep Disord Treat Care. 2014;3(4):15.

18. Sadeghniiat-Haghighi K, Montazeri A, Khajeh-Mehrizi A, Aminian O, Rahimi- Golkhandan A, Saraei M, et al. The Epworth Sleepiness Scale: translation and validation study of the Iranian Version. Sleep Breath. 2013;17(2)419-26. 
19. Udwadia ZF, Doshi AV, Lonkar SG, Singh CI. Prevalence of sleepdisordered breathing and sleep apnea in middle-aged urban Indian men. Am J Respir Crit Care Med. 2004;169(2):168-173.

20. Chung F, Subramanyam R, Liao P, Sasaki E, Shapiro C, Sun Y.High STOP-Bang score indicates a high probability of obstructive sleep apnoea. Br J Anaesth. 2012;108(5):768-75.

21. Silva GE, Vana KD, Goodwin JL, Sherrill DL, Quan SF. Identification of patients with sleep disordered breathing: comparing the fourvariable screening tool, STOP, STOP-Bang, and Epworth Sleepiness Scales. J Clin Sleep Med. 2011;7(5):467-72.

22. Ahmadi N, Chung SA, Gibbs A, Shapiro CM. The Berlin questionnaire for sleep apnea in a sleep clinic population: relationship to polysomnographic measurement of respiratory disturbance. Sleep Breath. 2008;12(1):39-45.

23. Johns MW. A new method for measuring daytime sleepiness: the Epworth sleepiness scale. Sleep. 1991;14(6):540-45.

24. Osman EZ, Osborne J, Hill PD, Lee BW.The Epworth Sleepiness Scale: can it be used for sleep apnoea screening among snorers? Clin Otolaryngol Allied Sci. 1999 Jun;24(3):239-41. 